\title{
Emergency Dispatch Center-Three Tactical Dilemmas
}

\author{
Rotaru LT* \\ University of Medicine and Pharmacy Craiova, Romania \\ *Corresponding author: Luciana Teodora Rotaru, University of Medicine and \\ Pharmacy Craiova, Romania, Tel: +40722702989; Email: lucianarotaru@yahoo.com
}

\begin{tabular}{c} 
Editorial \\
Volume 2 Issue 3 \\
Received Date: June 19, 2019 \\
Published Date: June 26,2019 \\
DoI: $10.23880 /$ jqhe-16000125 \\
\hline
\end{tabular}

\section{Editorial}

An integrated emergency dispatch is a specialized structure. It receives calls through emergency numbers, select, alerts and coordinates all the intervention crews of specialized intervention services, medical and nonmedical, responsible for solving crises. Thus viewed, an emergency dispatch is the revolving board for the management of informational and operational resources in pre-hospital emergency intervention and an important factor in medical patient's management and their flux dispersion to Emergency Departments.

A continuous increase in the number and complexity of cases with which emergency medical services must cope warrants the development of effective and efficient management tools to manage patient flow.

Besides the structural extension of emergency dispatch centers, and the possibility of increasing (however limited) the volume of staff in these services, the most critical tool of emergency dispatching remain flexibility in decision-making, intelligent application of medical practice guidelines and procedures for actions, and, in particular, the stratification of patients according to risk and severity criteria, allowing for a gradual and standardized allocation of resources within reasonable intervals, without aggravating their condition.

Since 1975, starting when Phoenix (Arizona) Fire Department empowered paramedics to interrogate callers and prioritize emergency calls, three development philosophies have generated evolutionary trends, and also dilemmas, that have marked the progress of emergency dispatch centers.

In 1976, when Dr. Jeff Clawson, a physician employed as Medical Director of the Salt Lake City Firefighters, developed a number of key questions to be used in processing emergency calls, and pre-arrival instructions and priorities for allocating resources. That is what generated the first dilemma: Professional dispatcher, or a field professional acting as a dispatcher?.

With the increase in the complexity of the activity, there was diversification of protocols for allocating medical intervention resources and communication and IT infrastructure (database, event localization, caller localization, resource localization, folders with multistage operational or medical support information were diversified. The dispatching activity was specialized, diversified, and integrated in multiple ways. The selection and training of a dedicated personnel for dispatching activity, as well as a staffing arrangement in which field personnel work in rotational shifts in dispatch, require special professionalism.

The partisans of the use in dispatch of medical assistants from the field (pre-hospital or ED) support the superior accuracy of assessing the seriousness of reported situations and the timely and practical transmission of medical advice to witnesses event. However, their difficulties are related to the overall image of the functioning of the system (poor compliance with the resource allocation protocols according to the amendments for each level of dispatch, resource cooperation, database management, communication). On the other hand, professional dispatchers without a field medical background are more adept at rigid following work procedures, but less efficient in extracting useful medical data that could influence the choice of a crew with a different level of competence.

In 1976, Professor Lareng validated a 20-year-old practice in Toulouse, France, and founded the SAMU (emergency services medical aid). He empowered 


\section{Journal of Quality in Health Care \& Economics}

physicians (most often from the base hospital of the reanimation teams) by allocating them the responsibility for the supervision of the entire medical dispatching. This created the second dilemma: does medical regulation also apply to dispatch, or only to medical procedures?

Are highly trained professional medical resources too expensive and too few to be placed in a dispatch center? Should they be placed such that they can directly attend to patients? Or will the presence of a medical doctor in a medical dispatch center ensure the allocation of this too rare and too expensive medical resource exactly to those patients who have the most urgent need for them? Further, telemedicine (especially with video transmission) does not assure exactly link that showing that high qualified Emergency Medicine physician presence into the integrated dispatch center is mandatory?.

Since 2000, Romania has been building an integrated emergency medical system model. In the Romanian vision, the evolution of emergency dispatch centers occurs on two levels: integration and specialization. In the following decade, the dispatching of all medical emergency services, first aid, fire \& rescue, police, have progressed through successive stages of integration and operational, structural, educational, communication, and procedural harmonization. This was when the third dilemma was born: fully integrated dispatch or integration of the dispatch centers?.
This is how emergency dispatch centers, born out of necessity during a crisis, have evolved to prevent crisis stemming from the overcrowding of pre-hospital emergency services.

That is why, when all is considered, there is no perfect dispatch model, but only systems better suited to specific organizations and various medical emergency systems worldwide, which evolve with conceptual, structural, and functional development.

The more differentiated the skills of the field medical crews, (first AID, medical crews with nurses, medical crews with GP, and even with more skilled medical crews - HEMS, mobile ICU), the more acute the need to refine the criteria for assessing the cases priority level at the allocated resource's competence, so the higher specialization of the medical decision.

The more extensive the territorial administrative responsibility of dispatch center is, the more refined the medical regulator competence and specialization has to be, and the more flexible the procedure needs to be.

The more diverse the resources, the higher integration level the dispatch center needs to be to ensure the accuracy, firmness and timeliness of every single order dispatch.

Are all these the answers to the three dilemmas? Or is this question itself the next dilemma? 